ample, scarcely hope to avoid using the horse-power as well as the watt), but in electrical work there is no need to give an arbitrary value to more than one quantity in addition to the three mechanical ones of length, mass and time. In the M.K.s. system the quantity selected for permeability is $10^{7}$ that of a vacuum. This automatically causes the pormittivity of' a vacuum to be $1.113 \times 10^{-10}$ units. Arbitrarily to make the permittivity of a vacuum one unit as is done in the e.s.u. system is to over-determine the units. It is very much to be hoped that this practice will be dropped, particularly as the exact conversion factor is not such a round figure as given in Dr. Guggenheim's table. It would only be so if the velocity of light were exactly $300,000 \mathrm{~km}$. per second which it is not.

Reginati O. KaPP.

Faculty of Engineering,

University College, London,

at University College, Swansea.

1 NATURE, 148, 751 (1941).

\section{Crystallization in an Inflated Rubber Balloon}

IT is well known that an oriented crystallization is produced in rubber by stretching. The rubber can be made to crystallize also by a two-dimensional homogeneous dilatation, as in the skin of an inflated ticular specimen; $b$ is the fibre axis indicating the direction of the main valency chain.

With this assumption, we interpret the result represented by Figs. 1 and 2 as follows. The dilatation of the rubber skin produces an oriented crystallization, the orientation being brought about by a tendency of the crystallites to arrange themselves: with the $b-c$-plane in the plane of the skin, that is, with the shorter $a$-axis perpendicular to the skin. The distribution in the plane around the normal is at random, giving as reflexions parts of Debye rings.

The effect is less marked in the thicker specimen, as will be seen when comparing Figs. 1 and 2. Fig. 3 is a photograph taken with the $\mathrm{X}$-ray beam passing through the centre of the sphere. This gives, of course, two sets of reflexions, from the near and the far side of the balloon respectively. The second one is scarcely visible in the reproduction. There are two Debye rings each with spacings equal to the (002) and (012) reflexions of which the latter is much stronger. The (200) reflexion is either completely absent or extremely weak. These findings appear to corroborate the proposition outlined above, which indeed is closely connected with what is known as 'higher orientation' in thin stretched. rubber bands.

Davy Faraday Research Laboratory,

Royal Institution, London, W.1.

${ }^{2}$ Lotmar and Meyer, Monatshefte, 69, 115 (1936).

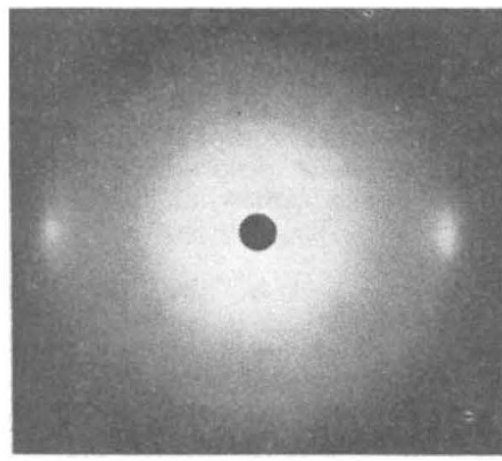

1

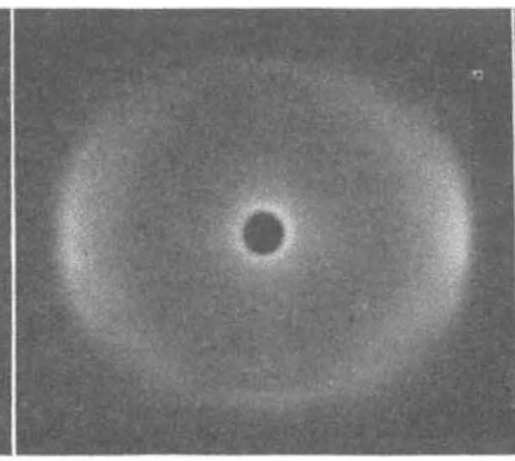

2

Thickness $0.6 \mathrm{~mm}$., inflated to $49 \mathrm{~mm}$. diameter. Distance $8 \mathrm{~cm}$., beam tangential.

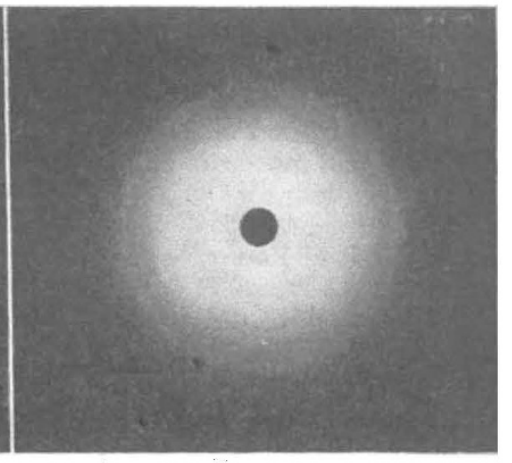

3

Thickness, $0 \cdot 1 \mathrm{~mm}$. , inflated to $31 \mathrm{~mm}$.
diameter. Distance, $8 \mathrm{~cm}$., beam tangential. X-RAY DIAGRAMS, TAKEN WITH UNFILTERED COPPER RADIATION, OF INFLATED RUDBER BALLOONS.

rubber balloon. I have experimented with small balloons of about $1 \mathrm{~cm}$. diameter when slack, made of pure vulcanized latex by dipping. When a balloon like this is inflated to $3-4 \mathrm{~cm}$. diameter and an X-ray beam strikes the sphere tangentially, photographs result as shown in Figs. 1 and 2, taken with balloons approximately 0.1 and $0.6 \mathrm{~mm}$. thick when slack. The spacings given by the reflexions in the horizontal plane, which contains the centre of the sphere, is $4.22 \mathrm{~A}$.; those of the short ares at top and bottom are $4.95 \mathrm{~A}$. and $6 \cdot 24 \mathrm{~A}$., corresponding to the (200), (012) and (002) reflexions in the ordinary fibre diagram which was taken separately. The arcs are blurred as they originate on an extended area of the sphere. The sharpness of the horizontal reflexions, on the other hand, is enhanced by a focusing effect.

Accepting a monoclinic structure with an angle $\beta=83^{\circ} 20^{\prime}$ as proposed by Lotmar and Meyer ${ }^{1}$, the dimensions of the elementary cell work out as $a=$ 8.5 A., $b=8 \cdot 2$ A. and $c=12 \cdot 6$ A. for this par-

\section{A Reversible Discharge Tube}

IN a recent letter, Asundi, Singh and Singh ${ }^{1}$ have described effects observed with a so-called reversible discharge tube. The phenomena recorded appear to be most easily explained by assuming that the discharge tube leaked slightly. This would account for the appearance of the hydrogen spectrum when the tube was freshly swept out with hydrogen, the appearance of nitrogen bands after standing, and the reappearance of the hydrogen spectrum on continuous running, the nitrogen being cleaned up by the alumin: ium electrodes, a well-known effect which is commonly made use of in hydrogen discharge tubes. The cleaning up would not occur so readily with external electrodes, and hence the discharge tube would be expected to continue to show nitrogen bands.

Chemical Technology Department, A. G. Gaydon.

Tondon, S.W.7.

1 NATURE, 149, 22 (1942). 